\title{
Exposure-background duality in the searches of neutrinoless double beta decay
}

\author{
M. K. Singh, ${ }^{1,2}$ H. T. Wong $\odot,{ }^{1, *}$ L. Singh, ${ }^{1,3}$ V. Sharma, ${ }^{1,2}$ V. Singh, ${ }^{1,2,3}$ and Q. Yue ${ }^{4}$ \\ ${ }^{1}$ Institute of Physics, Academia Sinica, Taipei 11529 \\ ${ }^{2}$ Department of Physics, Institute of Science, Banaras Hindu University, Varanasi 221005 \\ ${ }^{3}$ Department of Physics, School of Physical and Chemical Sciences, Central University of South Bihar, \\ Gaya 824236 \\ ${ }^{4}$ Department of Engineering Physics, Tsinghua University, Beijing 100084
}

(Received 27 August 2019; published 27 January 2020)

\begin{abstract}
Tremendous efforts are required to scale the summit of observing neutrinoless double beta decay $(0 \nu \beta \beta)$. This article quantitatively explores the interplay between exposure (target mass $\times$ data taking time) and background levels in $0 \nu \beta \beta$ experiments. In particular, background reduction can substantially alleviate the necessity of unrealistic large exposure as the normal mass hierarchy $(\mathrm{NH})$ is probed. The nondegenerate (ND)-NH can be covered with an exposure of $\mathcal{O}(100)$ ton-year, which is only an order of magnitude larger than those planned for next-generation projects-provided that the background could be reduced by $\mathcal{O}\left(10^{-6}\right)$ relative to the current best levels. It follows that background suppression will be playing increasingly important and investment-effective, if not determining, roles in future $0 \nu \beta \beta$ experiments with sensitivity goals of approaching and covering ND-NH.
\end{abstract}

DOI: $10.1103 /$ PhysRevD.101.013006

\section{INTRODUCTION}

The nature of the neutrinos [1] and, in particular, whether they are Majorana or Dirac particles, is an important problem in particle physics, the answer to which will have profound implications to the searches and formulation of physics beyond the Standard Model and the grand unified theories. Neutrinoless double beta decay $(0 \nu \beta \beta)$ is the most sensitive experimental probe to address this question [2]. Observation of $0 \nu \beta \beta$ implies (i) that neutrinos are Majorana particles, and (ii) lepton number violations. Since several decades, there are intense activities worldwide committed to the experimental searches of $0 \nu \beta \beta$.

Neutrino oscillation experiments [1,3] are producing increasingly precise information on the mass differences and mixings among the three neutrino mass eigenstates. The latest data imply slight preferences of the "normal hierarchy" (NH) over the "inverted hierarchy" (IH) in the structures of the neutrino mass eigenstates [4]. In parallel, cosmology data [5] provide stringent upper bounds on the total mass of the neutrinos, with good prospects on an actual measurement in the future. Together, a picture emerges providing a glimpse on the parameter space where

\footnotetext{
* Corresponding author. htwong@phys.sinica.edu.tw

Published by the American Physical Society under the terms of the Creative Commons Attribution 4.0 International license. Further distribution of this work must maintain attribution to the author(s) and the published article's title, journal citation, and DOI. Funded by SCOAP ${ }^{3}$.
}

positive observations of $0 \nu \beta \beta$ may reside. Experimental studies are expected to require significant effort and resources-especially so if NH is confirmed. Detailed quantitative studies on the optimal strategies "to scale this summit" with finite resources would be highly necessary.

The current work addresses one aspect of this issue. We studied the required exposures of $0 \nu \beta \beta$ projects vs the expected background $\mathrm{B}_{0}$ before the experiments are performed. The notations and formulation are described in Sec. II. The effects on the "discovery potentials" with varying $\mathrm{B}_{0}$, and the implied experimental strategies, are discussed in Sec. II B. The connections with the current landscape in neutrino physics are made in Sec. IIE via the choice of a particular model on the evaluation of nuclear matrix elements. Various aspects on the interplay between exposure and background in $0 \nu \beta \beta$ experiments are discussed in Sec. III A

Background typically includes two generic components each having different energy dependence-the ambient background and the irreducible intrinsic background from cosmogenic radioactivity and two-neutrino double beta decay $(2 \nu \beta \beta)$. Only the combined background is considered in this work, while ongoing research efforts are attending the different roles of the two components. In particular, the constraints imposed by the $2 \nu \beta \beta$ background to the detector resolution are discussed in Sec. III B.

\section{FORMULATIONS AND NOTATIONS}

\section{A. Double beta decay}

The process $0 \nu \beta \beta$ in candidate nucleus $A_{\beta \beta}$ refers to the decay 


$$
{ }_{Z}^{N} A_{\beta \beta} \rightarrow{ }_{Z+2}^{N-2} A+2 e^{-} .
$$

The experimental signature is distinctive. The summed kinetic energy of the two emitted electrons corresponds to a peak at the transition $\mathrm{Q}$ value $\left(Q_{\beta \beta}\right)$, which is known and unique for each $A_{\beta \beta}$.

The width of the $0 \nu \beta \beta$ peak (denoted by $\Delta$ in $\%$ ) characterizes the energy resolution of the detector, and is defined-a natural choice and also following convention in the literature for clarity-as the ratio of full-widthhalf-maximum (FWHM, denoted by $w_{1 / 2}$ ) to the total measurable energy $Q_{\beta \beta}$, such that $w_{1 / 2}=\left(\Delta \cdot Q_{\beta \beta}\right)$.

Various beyond-standard-model processes invoking lepton number violation can give rise to $0 \nu \beta \beta$ [6]. In the case of the "mass mechanism" where $0 \nu \beta \beta$ is driven by the Majorana neutrino mass, the $0 \nu \beta \beta$ half-life $\left(T_{1 / 2}^{0 \nu}\right)$ can be expressed by $[2,7]$

$$
\left[\frac{1}{T_{1 / 2}^{0 \nu}}\right]=G^{0 \nu} g_{A}^{4}\left|M^{0 \nu}\right|^{2}\left|\frac{\left\langle m_{\beta \beta}\right\rangle}{m_{e}}\right|^{2}
$$

where $m_{e}$ is the electron mass, $g_{A}$ is the effective axial vector coupling [8], $G^{0 \nu}$ is a known phase space factor [9] due to kinematics, and $\left|M^{0 \nu}\right|$ is the nuclear physics matrix element [10], while $\left\langle m_{\beta \beta}\right\rangle$ is the effective Majorana neutrino mass term which depends on neutrino masses $\left(m_{i}\right.$ for eigenstate $\left.\nu_{i}\right)$ and mixings $\left(U_{e i}\right.$ for the component of $\nu_{i}$ in $\nu_{e}$ ):

$$
\left\langle m_{\beta \beta}\right\rangle=\left|U_{e 1}^{2} m_{1}+U_{e 2}^{2} m_{2} e^{i \alpha}+U_{e 3}^{2} m_{3} e^{i \beta}\right|
$$

where $\alpha$ and $\beta$ are the Majorana phases.

The measurable half-life $T_{1 / 2}^{0 \nu}$ from an experiment which observes $N_{\mathrm{obs}}^{0 \nu}$ counts of $0 \nu \beta \beta$ events in time $t_{\mathrm{DAQ}}$ in a "region of interest" (RoI) at an efficiency of $\varepsilon_{\text {RoI }}$ can be expressed as

$$
T_{1 / 2}^{0 \nu}=\ln 2 \cdot N\left(A_{\beta \beta}\right) \cdot t_{\mathrm{DAQ}} \cdot\left[\frac{\varepsilon_{\mathrm{RoI}}}{N_{\mathrm{obs}}^{0 \nu}}\right]
$$

where $N\left(A_{\beta \beta}\right)$ is the number of $A_{\beta \beta}$ atoms being probed.

For simplicity in discussions and to allow the results be easily convertible to different configurations, while capturing the essence of the physics, results in this article are derived in the special "ideal" case where the target is made up of completely enriched $A_{\beta \beta}$ isotopes. That is, the isotopic abundance (IA) is $100 \%$. In additional, the various experimental efficiency factors are all unity $\left(\varepsilon_{\text {expt }}=100 \%\right)$. Accordingly, Eq. (4) becomes

$$
T_{1 / 2}^{0 \nu}=\ln 2 \cdot\left[\frac{N_{A}}{M\left(A_{\beta \beta}\right)}\right] \cdot \Sigma \cdot\left[\frac{\varepsilon_{\mathrm{RoI}}}{N_{\mathrm{obs}}^{0 \nu}}\right]
$$

where $N_{A}$ is Avogadro's number, $M\left(A_{\beta \beta}\right)$ is the molar mass of $A_{\beta \beta}$, and $\Sigma$ denotes the combined exposure (mass $\times t_{\mathrm{DAQ}}$ ) expressed in units of ton-year (ton-yr) at $A_{\beta \beta}$ at IA $=100 \%$ and $\varepsilon_{\text {expt }}=100 \%$. Effects due to these parameter choices and other assumptions will be discussed in Sec. II D where conversion relations to those for realistic experiments are given.

The expression of Eq. (5) applies to experiments with counting analysis. More sophisticated statistical methods are usually adopted to extract full information from a given data set. These typically exploit the energy spectral shapes, which are known for the signal and are predictable with uncertainties for the background. However, in the conceptual-design and sensitivity-projection stages of experiments, the simplified and intuitive approach of Eq. (5) will suffice, especially so in the low count rate Poisson statistics regime which is of particular interest in this article.

Combining the theoretical and experimental descriptions of $T_{1 / 2}^{0 \nu}$ from, respectively, Eqs. (2) and (5) gives

$$
\begin{aligned}
\left|M^{0 \nu}\right|^{2}\left[g_{A}^{4} \cdot H^{0 \nu}\right] & =\frac{1}{\left\langle m_{\beta \beta}\right\rangle^{2}}\left[\frac{1}{\Sigma} \cdot \frac{N_{\mathrm{obs}}^{0 \nu}}{\varepsilon_{\mathrm{RoI}}}\right], \quad \text { where } \\
H^{0 \nu} & \equiv \ln 2\left[\frac{N_{A}}{M\left(A_{\beta \beta}\right) \cdot m_{e}^{2}}\right] G^{0 \nu}
\end{aligned}
$$

is called "specific phase space" in the literature [7].

\section{B. Discovery potential}

In our context, $\mathrm{B}_{0}$ is expected background counts within the RoI around $Q_{\beta \beta}$. This can, in principle, be predicted with good accuracies prior to the experiments. The sensitivity goals of experiments are typically expressed in the literature [11] as: "Discovery potential at $3 \sigma$ with $50 \%$ probability" $\left(\mathrm{P}_{50}^{3 \sigma}\right)$ and "upper limits at $90 \%$ confidence level," which characterize possible positive and negative outcomes, respectively. We focus on $\mathrm{P}_{50}^{3 \sigma}$ in this work, for the reason that next-generation $0 \nu \beta \beta$ experiments should be designed to have the maximum reach of discovery, rather than setting limits.

Poisson statistics is necessary to handle low background and rare signal processes. The dependence of the required average signal $\left(\mathrm{S}_{0}\right)$ vs $\mathrm{B}_{0}$ under $\mathrm{P}_{50}^{3 \sigma}$ and other discovery potential criteria are depicted in Fig. 1(a). For a given real and positive $\mathrm{B}_{0}$ as input and using $\mathrm{P}_{50}^{3 \sigma}$ as illustration, the Poisson distribution $P(i ; \mu)$ is constructed with mean $\mu=\mathrm{B}_{0}$. The observed count $N_{\mathrm{obs}}^{3 \sigma}$ is evaluated as the smallest integer which satisfies

$$
\sum_{i=0}^{N_{\text {obs }}^{3 \sigma}} P\left(i ; \mathrm{B}_{0}\right) \geq(1-0.00135)
$$


(a)

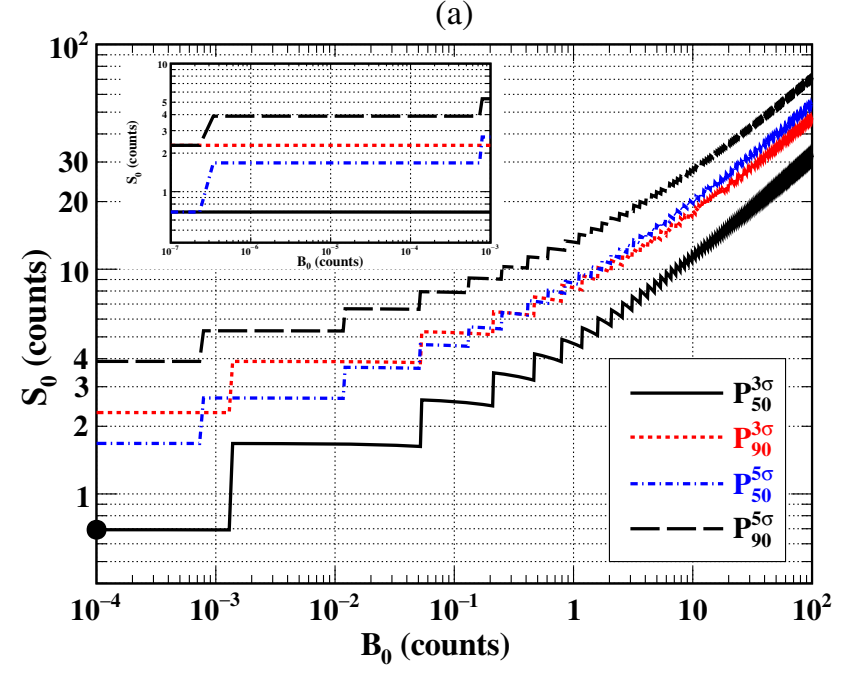

(b)

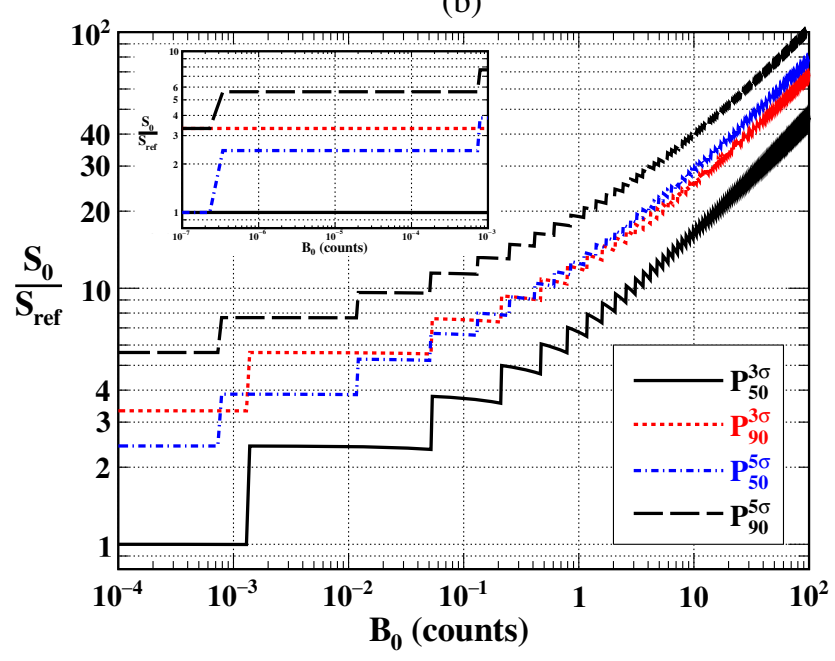

FIG. 1. The variations of (a) $\mathrm{S}_{0}$ and (b) ratios of $\mathrm{S}_{0}$ to $\mathrm{S}_{\text {ref }}$ vs $\mathrm{B}_{0}$ in the discovery potential of $\geq 3 \sigma, 5 \sigma$ with $\geq 50 \%, 90 \%$. The specific case of $\mathrm{P}_{50}^{3 \sigma}$ at $3 \sigma$ and $50 \%$ is adopted as the criteria in this work. The level of $\mathrm{S}_{\text {ref }}$ at the background-free condition for $\mathrm{P}_{50}^{3 \sigma}$ is represented with a black dot in (a).

where 0.00135 is the fraction of a Gaussian distribution in the interval $[+3 \sigma, \infty]$. This is the minimal observed event integer number with $\geq 3 \sigma$ significance over a predicted average background $B_{0}$. The output $S_{0}$ is the minimal signal strength corresponding to the case where the average total event $\left(\mathrm{B}_{0}+\mathrm{S}_{0}\right)=N_{\text {obs }}^{3 \sigma}$ with $\geq 50 \%$ probability. This is evaluated as the minimum value which satisfies another Poisson distribution under the condition:

$$
\sum_{i=0}^{N_{\text {obs }}^{3 \sigma}} P\left(i ;\left[\mathrm{B}_{0}+\mathrm{S}_{0}\right]\right) \geq 0.5 .
$$

It can be inferred from Fig. 1(a) that the "backgroundfree" level with $\mathrm{P}_{50}^{3 \sigma}$ criteria corresponds to a background of $\mathrm{B}_{0}<10^{-3}$ and a reference-point signal of $\mathrm{S}_{0} \equiv$ $\mathrm{S}_{\mathrm{ref}}=0.69$.

The ratios of $S_{0}$ relative to $S_{\text {ref }}$ are depicted in Fig. 1(b). It can be seen that one would require factors of 6.7(16.8) stronger signals to establish positive results at $\mathrm{P}_{50}^{3 \sigma}$ when $\mathrm{B}_{0}$ increases from $<10^{-3}$ to $1(10)$.

While the predicted average background $\mathrm{B}_{0}$ can be continuous and real numbers, only integer counts can be observed in an experiment. This gives rise to the relations being inequalities in Eqs. (7) and (8) and consequently the steps in Figs. 1(a) and 1(b). In addition, signal and background events are indistinguishable experimentally. The $\mathrm{P}_{50}^{3 \sigma}$ criteria is applied to $\left(\mathrm{B}_{0}+\mathrm{S}_{0}\right)$ vs $\mathrm{B}_{0}$, while the $\mathrm{S}_{0}$ dependence on $B_{0}$ is shown in Figs. 1(a) and 1(b). This is the origin of the negative slopes in various segments.

\section{Background index}

The theme of this work is to study the interplay between required exposure and background in $0 \nu \beta \beta$ experiments to meet certain $\left\langle m_{\beta \beta}\right\rangle$ target sensitivities.

In realistic experiments, it is more instructive to characterize background with respect to exposure and the RoI energy range, such that the relevant parameter is the "background index" (BI) defined as

$$
\mathrm{BI} \equiv \frac{\mathrm{B}_{0}(\mathrm{RoI})}{\Sigma}
$$

which is the background within the RoI (chosen to be $\equiv w_{1 / 2}$, following convention) per 1 ton-year of exposure, with dimension [counts $/\left(w_{1 / 2}\right.$-ton-yr $)$ ]. Background levels expressed in BI are universally applicable to compare sensitivities of varying $A_{\beta \beta}$ in different experiments.

\section{Conversion to realistic configurations}

As explained in Sec. II A, the (BI, $\Sigma)$ results presented in this article correspond to the ideal case where IA $=100 \%$ and $\varepsilon_{\text {expt }}=100 \%$. In addition, while the range of $g_{A} \in$ $[0.6,1.27]$ is generally considered possible $[7,8]$, the "unquenched" free nucleon value of $g_{A}=1.27$ is adopted.

The required exposure $\left(\Sigma^{\prime}\right)$ in realistic experiments would be larger and can be readily converted from the $\Sigma$ values via

$$
\Sigma^{\prime} \simeq \Sigma \cdot \frac{1}{\mathrm{IA}} \cdot \frac{1}{\varepsilon_{\mathrm{expt}}} \cdot W_{\Sigma}\left(g_{A}\right),
$$

where $W_{\Sigma}\left(g_{A}\right)$ is the weight factor for $\Sigma$ due to the $g_{A}$ dependence [10,12] of $T_{1 / 2}^{0 \nu}$ in Eq. (2), relative to the values at $g_{A}=1.27$. It is depicted in Fig. 3 for the case of ${ }^{76} \mathrm{Ge}$. The finite band width as a function of $g_{A}$ is the consequence of the spread in $\left|M^{0 \nu}\right|^{2}$ predictions $[10,12]$. The specific case where $\left|M^{0 \nu}\right|^{2}$ is independent of $g_{A}$ implies $\Sigma \propto\left[g_{A}\right]^{-4}$ and is denoted by the dotted line. 
TABLE I. Summary of the key parameters used in this work. Inputs are the $\mathrm{IH}$ and $\mathrm{NH}$ bands at $\pm 3 \sigma$ of the ND scenario at $m_{\min }<10^{-4} \mathrm{eV}$ from existing measurements [1], such that $\left\langle m_{\beta \beta}\right\rangle_{-}<\left\langle m_{\beta \beta}\right\rangle<\left\langle m_{\beta \beta}\right\rangle_{+}$. The posterior $\left\langle m_{\beta \beta}\right\rangle_{95 \%}$ denotes the 95\% lower bound for the $\left\langle m_{\beta \beta}\right\rangle$ distribution, taking an uncorrelated $(\alpha, \beta)$ and the uncertainty range in $\left|M^{0 \nu}\right|^{2}$ as prior [11]. The corresponding minimal exposures at background-free levels under criteria $\mathrm{P}_{50}^{3 \sigma}$ are given as $\Sigma_{\min }$. The reduction fraction in $\Sigma$ from $\left\langle m_{\beta \beta}\right\rangle_{-}$to $\left\langle m_{\beta \beta}\right\rangle_{95 \%}$ is denoted by $f_{95 \%}$. The values of $\left(\left\langle m_{\beta \beta}\right\rangle_{-},\left\langle m_{\beta \beta}\right\rangle_{+}\right)$and $\left\langle m_{\beta \beta}\right\rangle_{95 \%}$ define the IH/NH band width and dotted lines, respectively, in Figs. 4, 5, and 8 in this article.

\begin{tabular}{lcccc}
\hline \hline & $\left\langle m_{\beta \beta}\right\rangle_{-}$ & $\left\langle m_{\beta \beta}\right\rangle_{+}$ & $\left\langle m_{\beta \beta}\right\rangle_{95 \%}$ & $f_{95 \%}$ \\
\hline $\mathrm{IH}:$ & & & & \\
$\left\langle m_{\beta \beta}\right\rangle\left(\times 10^{-3} \mathrm{eV}\right)$ & & & & \\
& 14 & 51 & 20 & $\ldots$ \\
$\Sigma_{\min }($ ton-yr $)$ & 1.7 & 0.13 & 0.83 & 0.49 \\
$\mathrm{NH}:$ & & & & \\
$\left\langle m_{\beta \beta}\right\rangle\left(\times 10^{-3} \mathrm{eV}\right)$ & & & & \\
& 0.78 & 4.3 & 3.0 & $\ldots$ \\
$\Sigma_{\text {min }}$ (ton-yr) & 550 & 18 & 37 & 0.068 \\
\hline \hline
\end{tabular}

The background index defined relative to $\Sigma^{\prime}$ for realistic configurations can accordingly be expressed as

$$
\mathrm{BI}^{\prime}\left(\Sigma^{\prime}\right) \simeq \mathrm{BI} \cdot\left[\frac{\Sigma}{\Sigma^{\prime}}\right]
$$

such that $\Sigma^{\prime}>\Sigma$ and $\mathrm{BI}^{\prime}<\mathrm{BI}$. Realistic experiments naturally imply larger exposure and more stringent background requirements.

\section{E. Neutrino physics connections}

Results from neutrino oscillation experiments $[1,3]$ indicate that the $m_{i}$ of the three active $\nu_{i}$ have structures corresponding to either IH or NH. The values of $\left\langle m_{\beta \beta}\right\rangle$ are constrained and depend on the absolute neutrino mass scale, and are typically expressed in terms of the lowest mass eigenstates $m_{\min }$. The $\pm 3 \sigma$ ranges of $\left\langle m_{\beta \beta}\right\rangle$ with the nondegenerate (ND) mass eigenstate scenarios at $m_{\min }<$ $10^{-4} \mathrm{eV}$, denoted by $\left\langle m_{\beta \beta}\right\rangle_{-}$and $\left\langle m_{\beta \beta}\right\rangle_{+}$, are constant and listed in Table I.

There are no experimental constraints on the Majorana phases $(\alpha, \beta)$. It is, in principle, possible to have accidental cancellation which leads to very small $\left\langle m_{\beta \beta}\right\rangle$ at $m_{\min } \in[1,10] \times 10^{-3} \mathrm{eV}$. However, under the reasonable assumption that they are uncorrelated and have uniform probabilities within $[0,2 \pi]$, a posterior probability distributions of $\left\langle m_{\beta \beta}\right\rangle$ can be assigned [11]. The 95\% lower limit, denoted as $\left\langle m_{\beta \beta}\right\rangle_{95 \%}$ and listed in Table I, shows that the vanishing values of $\left\langle m_{\beta \beta}\right\rangle$ are disfavored.

The current generation of oscillation experiments may reveal nature's choice between the two hierarchy options.

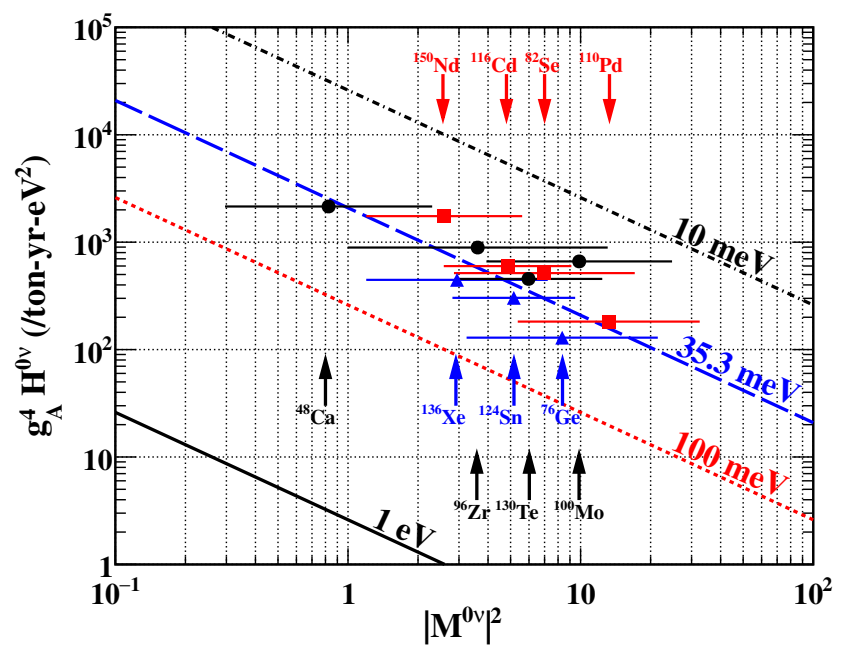

FIG. 2. Variations of "specific phase space" $g_{A}^{4} H^{0 \nu}$, as defined in Eq. (6), vs $\left|M^{0 \nu}\right|^{2}$ for various $A_{\beta \beta}$. The geometric mean of the range of $\left|M^{0 \nu}\right|^{2}$ is presented as the data points. The best-fit and other diagonal lines correspond to the matching $\left\langle m_{\beta \beta}\right\rangle$ values at $g_{A}=1.27$ that give rise to a $0 \nu \beta \beta$ rate of 1 event per ton-year at full efficiency. This formulation is adopted from Figs. 2 and 3 of Ref. [7].

In particular, there is an emerging preference of $\mathrm{NH}$ over $\mathrm{IH}$ $[1,4]$. Moreover, the combined cosmology data may provide a measurement on the sum of $m_{i}$ [5]. Therefore, it can be expected that the ranges of parameter space of $\left\langle m_{\beta \beta}\right\rangle$ in $0 \nu \beta \beta$ searches will be further constrained.

Extracting neutrino mass information via Eq. (2) from the experimentally measured $T_{1 / 2}^{0 \nu}$ requires knowledge of $\left|M^{0 \nu}\right|^{2}$ and $g_{A}$. There are different schemes to calculate

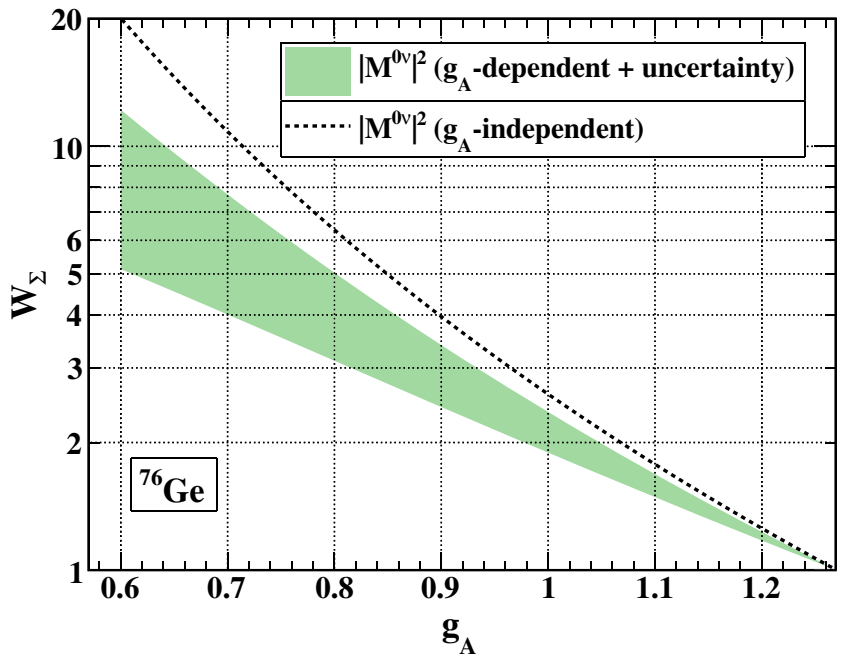

FIG. 3. Variation of $W_{\Sigma}$ as defined in Eq. (10) due to changes in $g_{A}$ relative to that of $g_{A}=1.27$ in the case of ${ }^{76} \mathrm{Ge}$. The finite band width is the consequence of the spread in $\left|M^{0 \nu}\right|^{2}$ predictions $[10,12]$. The specific case where $\left|M^{0 \nu}\right|^{2}$ is independent of $g_{A}$ such that $\Sigma \propto\left[g_{A}\right]^{-4}$ is denoted by the dotted line. 


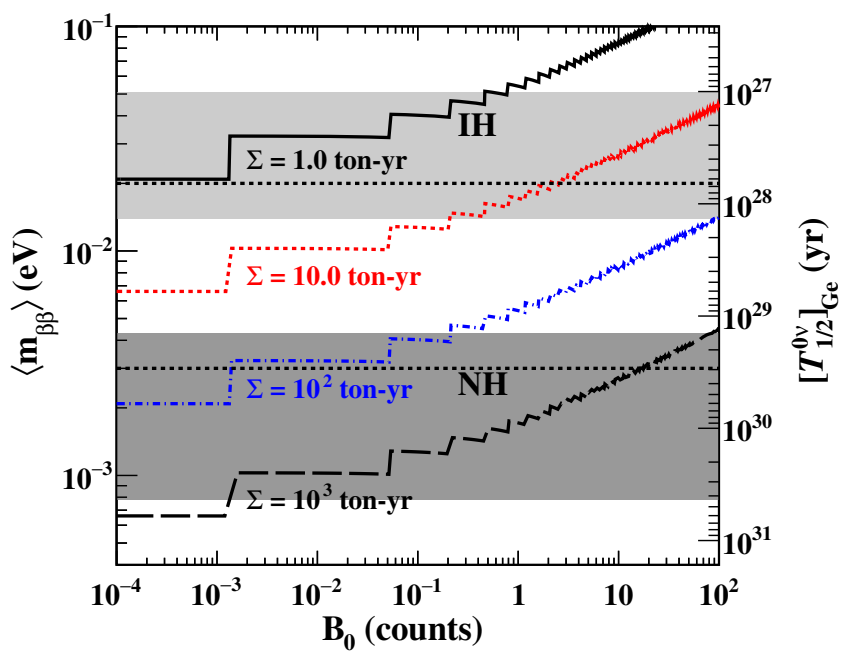

FIG. 4. The variation of $\left\langle m_{\beta \beta}\right\rangle$ with $\mathrm{B}_{0}$ following $\mathrm{P}_{50}^{3 \sigma}$, with $\mathrm{RoI}=w_{1 / 2}$ for ${ }^{76} \mathrm{Ge}$ at $\Sigma=(1 ; 10 ; 100 ; 1000)$ ton-yr following Eq. (6) at $g_{A}=1.27$. The IH $(\mathrm{NH})$ bands are defined by $\left(\left\langle m_{\beta \beta}\right\rangle_{-},\left\langle m_{\beta \beta}\right\rangle_{+}\right)$, while their $\left\langle m_{\beta \beta}\right\rangle_{95 \%}$ values are denoted as dotted lines. The corresponding variations of $\left[T_{1 / 2}^{0 \nu}\right]_{\mathrm{Ge}}$ vs $\mathrm{B}_{0}$ are displayed as the right vertical axis. This is specific to ${ }^{76} \mathrm{Ge}$, while the equivalent values for other $A_{\beta \beta}$ can be derived via Eq. (13).

$\left|M^{0 \nu}\right|^{2}$ for different $A_{\beta \beta}$ [10]. Deviations among their results are the main contributors to the theoretical uncertainties. Another source of uncertainties is the values of $g_{A}$, which may differ between a free nucleon and complex nuclei [8].

Studies of Ref. [7] suggest that, in the case where $0 \nu \beta \beta$ is driven by the neutrino mass mechanism, there exists an

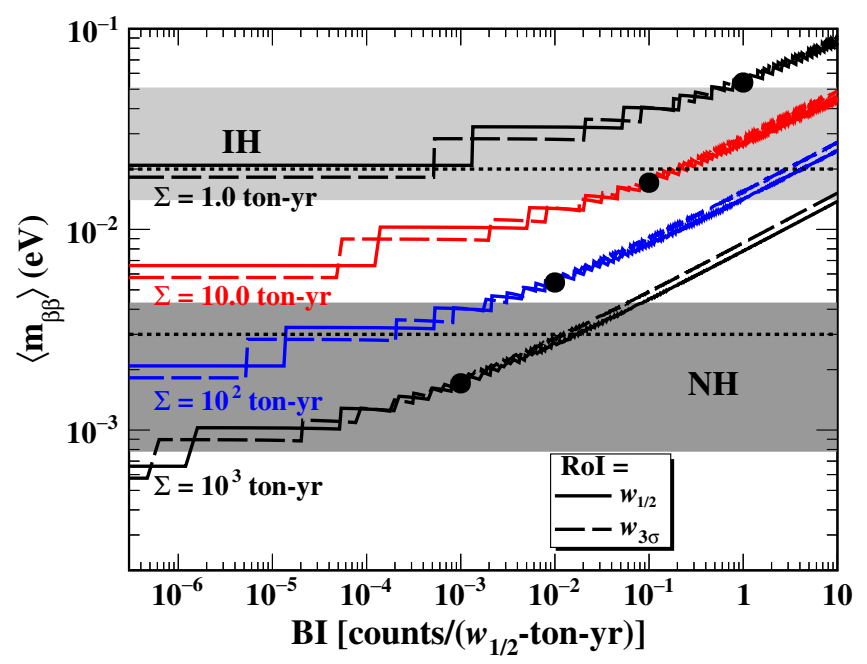

FIG. 5. Sensitivities of $\left\langle m_{\beta \beta}\right\rangle$ vs BI, as defined in Eq. (9) which is universally applicable to all $A_{\beta \beta}$, following $\mathrm{P}_{50}^{3 \sigma}$ under different exposures at $\Sigma=(1 ; 10 ; 100 ; 1000)$ ton-yr. The IH $(\mathrm{NH})$ bands are defined by $\left(\left\langle m_{\beta \beta}\right\rangle_{-},\left\langle m_{\beta \beta}\right\rangle_{+}\right)$, while their $\left\langle m_{\beta \beta}\right\rangle_{95 \%}$ values are denoted as dotted lines. Both options of $\mathrm{RoI}=w_{1 / 2}$ and $w_{3 \sigma}$ are displayed. Black dots correspond to the benchmark $\left[1\right.$ count $\left./\left(w_{1 / 2}-\Sigma\right)\right]$ background levels. (a)

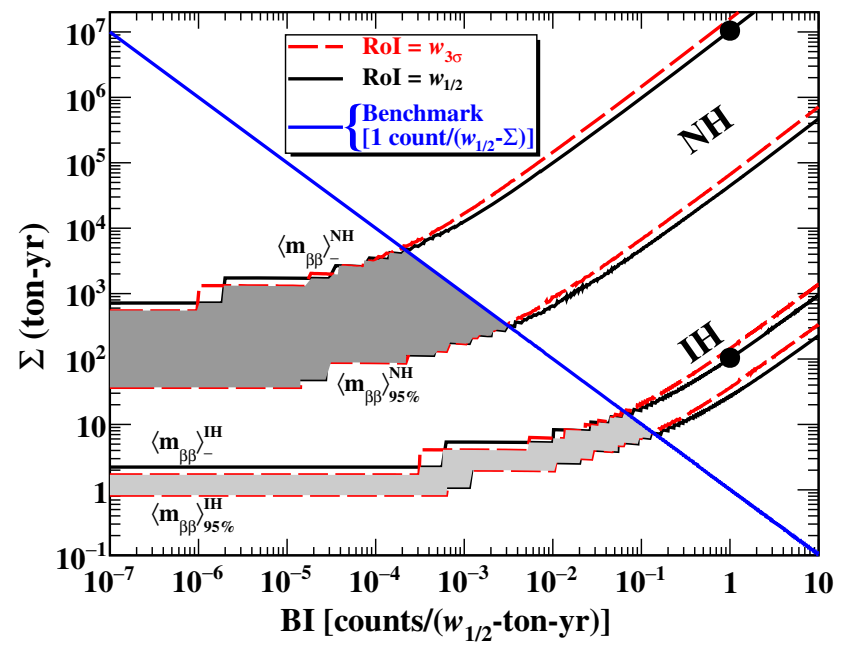

(b)

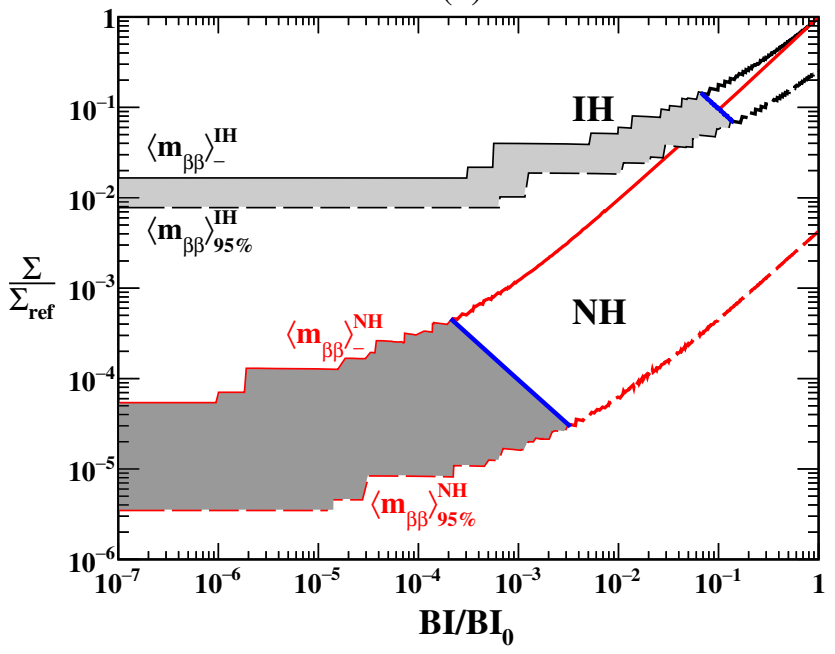

FIG. 6. (a) Required exposure universally applicable to all $A_{\beta \beta}$ to cover $\left\langle m_{\beta \beta}\right\rangle_{95 \%}$ and $\left\langle m_{\beta \beta}\right\rangle_{-}$under $\mathrm{P}_{50}^{3 \sigma}$ at $\mathrm{IH}$ and $\mathrm{NH}$ vs BI. The benchmark background [1 count $\left./\left(w_{1 / 2}-\Sigma\right)\right]$ condition is superimposed as the blue contour. The shaded regions correspond to the preferred hardware specification space for future $0 \nu \beta \beta$ experiments. (b) The relation between exposure and background reduction relative to the current $\mathrm{BI}_{0}=1$ count/ $\left(w_{1 / 2}\right.$-ton-yr $)$ and its corresponding required exposures $\Sigma_{\text {ref }}^{\mathrm{IH}(\mathrm{NH})}=110$ ton-yr(11 Mton-yr) for IH (NH). The reference points $\left(\mathrm{BI}_{0}, \Sigma_{\text {ref }}^{\mathrm{IH}(\mathrm{NH})}\right)$ are represented by black dots in (a). Sensitivities with both $\mathrm{RoI}=w_{1 / 2}$ and $w_{3 \sigma}$ are displayed in (a), while the more sensitive of the two schemes are shown in (b). The shaded regions match those of (a).

inverse correlation between $G^{0 \nu}$ and $\left|M^{0 \nu}\right|^{2}$ in Eq. (2), the consequence of which is that the decay rates per unit mass for different $A_{\beta \beta}$ are similar at given $\left\langle m_{\beta \beta}\right\rangle$ and constant $g_{A}$. That is, there is no favored $0 \nu \beta \beta$ isotope from the nuclear physics point of view.

This empirical observation originates partially to the large uncertainties in $\left|M^{0 \nu}\right|^{2}$ and $g_{A}$. To derive numerical 
results which would shed qualitative insights without involving excessive discussions on the choice of $\left|M^{0 \nu}\right|^{2}$, we assume that this correlation is quantitatively valid.

We follow Ref. [7] in adopting the geometric means of the realistic ranges for the various $\left|M^{0 \nu}\right|^{2}$ in different isotopes. The data points can be parametrized by straight lines at given $\left\langle m_{\beta \beta}\right\rangle$, as depicted in Fig. 2. That is, $\left[\left|M^{0 \nu}\right|^{2}\left(g_{A}^{4} H^{0 \nu}\right)\right]$ is a constant at fixed $\left\langle m_{\beta \beta}\right\rangle$ independent of $A_{\beta \beta}$. The displayed $\left\langle m_{\beta \beta}\right\rangle$ values in Fig. 2 correspond to $0 \nu \beta \beta$ decay rates of $\left[N_{\mathrm{obs}}^{0 \nu} / \Sigma\right]=1 /$ ton-yr at $g_{A}=1.27$ and full efficiency. The best fit at this decay rate corresponds to $\left\langle m_{\beta \beta}\right\rangle=\left(35 \times 10^{-3}\right) \mathrm{eV}$.

Following Eq. (6), this model leads to a simplifying consequence that

$$
\Sigma(\text { ton-yr }) \cdot\left[\frac{\varepsilon_{\mathrm{RoI}}}{N_{\mathrm{obs}}^{0 \nu}}\right] \propto \frac{1}{\left\langle m_{\beta \beta}\right\rangle^{2}}
$$

at IA $=100 \%$ and $\varepsilon_{\text {expt }}=100 \%$, which is universally applicable to all $A_{\beta \beta}$. The proportional constant can be derived via the best-fit values of Fig. 2 .

Given a background $\mathrm{B}_{0}$ as input, the required $\mathrm{S}_{0}$ to establish a signal under $\mathrm{P}_{50}^{3 \sigma}$ can be derived via Fig. 1(a). This is related to the mean of $N_{\text {obs }}^{0 \nu}$ at known $\varepsilon_{\text {RoI }}$. Neutrino physics provides constraints on $\left\langle m_{\beta \beta}\right\rangle$ with several scales of interest given in Table I. The output values of $\Sigma$ and BI can be derived with Eqs. (12) and (9), respectively.

The $\Sigma$ values thus inferred in what follows could be interpreted with the typical uncertainties of a "factor of two, both directions" (that is, within a factor of [0.5, 2.0] of the nominal values) to match our current understanding of $\left|M^{0 \nu}\right|^{2}$.

\section{SENSITIVITY DEPENDENCE}

It is well-known, following Eqs. (2) and (5), that the sensitivity to $\left[1 /\left\langle m_{\beta \beta}\right\rangle\right]$ is proportional to $\Sigma^{\frac{1}{2}}$ as $\mathrm{B}_{0} \rightarrow 0$ and to $\Sigma^{\frac{1}{4}}$ at large $\mathrm{B}_{0}$. We further investigate the $\mathrm{B}_{0}$ dependence quantitatively and in the context of the preferred $\mathrm{IH}$ and $\mathrm{NH}$ ranges with the model of Ref. [7]. The specific $\left\langle m_{\beta \beta}\right\rangle$ values of Table I- $\left(\left\langle m_{\beta \beta}\right\rangle_{-},\left\langle m_{\beta \beta}\right\rangle_{+},\left\langle m_{\beta \beta}\right\rangle_{95 \%}\right)$ for both IH and $\mathrm{NH}-$ serve to provide reference scales.

\section{A. Required exposure and background}

The variations of $\left\langle m_{\beta \beta}\right\rangle$ vs $\mathrm{B}_{0}$ with different $\Sigma$ at $\mathrm{RoI}=$ $w_{1 / 2}$ (such that $\varepsilon_{\mathrm{RoI}} \simeq 76 \%$ ) under the criteria of $\mathrm{P}_{50}^{3 \sigma}$ are depicted in Fig. 4, with the $\mathrm{IH}$ and $\mathrm{NH}$ bands superimposed. The matching $T_{1 / 2}^{0 \nu}$ for ${ }^{76} \mathrm{Ge}$ is illustrated. The equivalent half-life sensitivities for other isotopes $A_{\beta \beta}$ can be derived via

$$
\left[T_{1 / 2}^{0 \nu}\right]_{A_{\beta \beta}}=\left[T_{1 / 2}^{0 \nu}\right]_{{ }^{6} \mathrm{Ge}}\left(\frac{76}{A_{\beta \beta}}\right) .
$$

The figure depicts how the same exposure can be used to probe longer $T_{1 / 2}^{0 \nu}$ and smaller $\left\langle m_{\beta \beta}\right\rangle$ with decreasing background.

The dependence of $\left\langle m_{\beta \beta}\right\rangle$ sensitivities to BI is depicted in Fig. 5. Taking RoI $=w_{1 / 2}$ is obviously not the optimal choice when the expected background $\mathrm{B}_{0} \rightarrow 0$. An alternative choice for low $\mathrm{B}_{0}$ is $\mathrm{RoI} \equiv w_{3 \sigma}$ covering $\pm 3 \sigma$ of $Q_{\beta \beta}$, such that $\varepsilon_{\text {RoI }} \cong 100 \%$. Both schemes are illustrated in Fig. 5. The choice of RoI $=w_{3 \sigma}$ at $\mathrm{B}_{0} \rightarrow 0$ would expectedly give better sensitivity by a factor of $\varepsilon_{\text {RoI }}\left(w_{1 / 2}\right)=0.76$, such that the covered $T_{1 / 2}^{0 \nu}$ is $32 \%$ longer, or the required $\Sigma$ is $24 \%$ less.

The required exposure to probe $\left\langle m_{\beta \beta}\right\rangle_{95 \%}$ and $\left\langle m_{\beta \beta}\right\rangle_{-}$ with both RoI selections in both $\mathrm{IH}$ and $\mathrm{NH}$ are depicted in Fig. 6(a). Superimposed as a blue contour is the "benchmark" background level at 1 count $/\left(w_{1 / 2}-\Sigma\right)$ where the first background event would occur at a given exposure. The benchmark level also represents the transition in the effectiveness of probing $\left\langle m_{\beta \beta}\right\rangle$ with increasing exposure. The shaded regions correspond to the preferred hardware specification space for future $0 \nu \beta \beta$ experiments-where

TABLE II. Required exposure $(\Sigma)$ to cover $\left\langle m_{\beta \beta}\right\rangle_{95 \%}^{\mathrm{IH}(\mathrm{NH})}$ and $\left\langle m_{\beta \beta}\right\rangle_{-}^{\mathrm{IH}(\mathrm{NH})}$ at different background scenarios in descending order of

\begin{tabular}{|c|c|c|c|c|c|c|}
\hline \multicolumn{3}{|c|}{ Background } & \multicolumn{4}{|c|}{ Required $\Sigma$ (ton-yr) to Cover } \\
\hline Scenario & & BI [counts $/\left(w_{1 / 2}-\right.$ ton-yr $\left.)\right]$ & $\left\langle m_{\beta \beta}\right\rangle_{95 \%}^{\mathrm{IH}}$ & $\left\langle m_{\beta \beta}\right\rangle_{-}^{Y H}$ & $\left\langle m_{\beta \beta}\right\rangle_{95 \%}^{\mathrm{NH}}$ & $\left\langle m_{\beta \beta}\right\rangle_{-}^{\mathrm{NH}}$ \\
\hline Best published [13] & & 1 & 27 & 110 & $4.4 \times 10^{4}$ & $11 \times 10^{6}$ \\
\hline Next generation projected [14] & \} & 0.1 & 6.1 & 19 & $4.7 \times 10^{3}$ & $0.97 \times 10^{6}$ \\
\hline \multirow[t]{4}{*}{ Benchmark $\left[1\right.$ count $\left./\left(w_{1 / 2}-\Sigma\right)\right]$} & $\mathrm{IH}:\{$ & 0.14 & 7.3 & $\cdots$ & $\ldots$ & $\ldots$ \\
\hline & & 0.067 & $\cdots$ & 15 & $\cdots$ & $\cdots$ \\
\hline & $\mathrm{NH}:\{$ & $3.1 \times 10^{-3}$ & $\cdots$ & $\cdots$ & 330 & $\ldots$ \\
\hline & & $2.2 \times 10^{-4}$ & $\cdots$ & $\cdots$ & $\cdots$ & $4.6 \times 10^{3}$ \\
\hline \multirow[t]{4}{*}{ "Background-Free" } & $\mathrm{IH}:\{$ & $\leq\left(6.3 \times 10^{-4}\right)$ & 0.83 & $\cdots$ & $\cdots$ & $\ldots$ \\
\hline & & $\leq\left(3.1 \times 10^{-4}\right)$ & 0.83 & 1.7 & $\cdots$ & $\cdots$ \\
\hline & $\mathrm{NH}:\{$ & $\leq\left(1.4 \times 10^{-5}\right)$ & 0.83 & 1.7 & 37 & $\ldots$ \\
\hline & & $\leq\left(0.96 \times 10^{-6}\right)$ & 0.83 & 1.7 & 37 & 550 \\
\hline
\end{tabular}
intensity. The BI values follow from Eq. (9). 
the exposure should be sufficient to cover at least $\left\langle m_{\beta \beta}\right\rangle_{95 \%}^{\mathrm{IH}(\mathrm{NH})}$, and there would be less than one background event per $w_{1 / 2}$ over the full exposure.

The required exposures under various background conditions are summarized in Table II. The best published background level is $1.0_{-0.4}^{+0.6}$ counts/(keV-ton-yr) or $\mathrm{BI} \sim 3$ counts $/\left(w_{1 / 2}\right.$-ton-yr $)$ from the GERDA experiment on ${ }^{76} \mathrm{Ge}$ [13]. For simplicity, the "best" current background is taken to be $\mathrm{BI} \equiv \mathrm{BI}_{0}=1$ count/ $\left(w_{1 / 2}\right.$-ton-yr $)$ in what follows. This background would correspond to $\Sigma_{\text {ref }}^{\mathrm{IH}(\mathrm{NH})}=110$ ton-yr(11 Mton-yr) to cover $\left\langle m_{\beta \beta}\right\rangle_{-}^{\mathrm{IH}}\left(\left\langle m_{\beta \beta}\right\rangle_{-}^{\mathrm{NH}}\right)$.

Such a large required exposure is inefficient and unrealistic, so that the background should be significantly reduced to allow the quest to advance. The target exposure is $\Sigma=10$ ton-yr for the next-generation $0 \nu \beta \beta$ projects to cover IH with a ton-scale detector target [14]. Following Fig. 5, this exposure would require $\mathrm{BI}<(0.21,0.033)$ counts $/\left(w_{1 / 2}\right.$-ton-yr $)$ to cover $\left(\left\langle m_{\beta \beta}\right\rangle_{95 \%},\left\langle m_{\beta \beta}\right\rangle_{-}^{\mathrm{IH}}\right)$. This matches the background specifications of $\mathrm{BI}=\mathcal{O}(0.1)$ counts $/\left(w_{1 / 2}\right.$-ton-yr).

The background-free $\left(\mathrm{BI}_{\min }\right)$ —equivalently, minimalexposure $\left(\Sigma_{\min }\right)$ - condition is where one single observed event can establish the signal at the $\mathrm{P}_{50}^{3 \sigma}$ criteria. Their values at the benchmark $\left\langle m_{\beta \beta}\right\rangle$ 's are given in Table I.

The choice of $\left\langle m_{\beta \beta}\right\rangle_{-}$to define $\Sigma$ is a conservative one. Since $\left.\left\langle m_{\beta \beta}\right\rangle_{95 \%}\right\rangle\left\langle m_{\beta \beta}\right\rangle_{-}$from Table I, the minimum exposure $\Sigma_{\min }$ corresponding to $\left\langle m_{\beta \beta}\right\rangle_{95 \%}$ is reduced relative to that for $\left\langle m_{\beta \beta}\right\rangle_{-}$by a fraction given as $f_{95 \%}$.

The variations of $\left(\mathrm{BI}_{\min }, \Sigma_{\text {min }}\right)$ with $\left\langle m_{\beta \beta}\right\rangle$ are depicted in Fig. 7. As shown by the black dots and also listed in

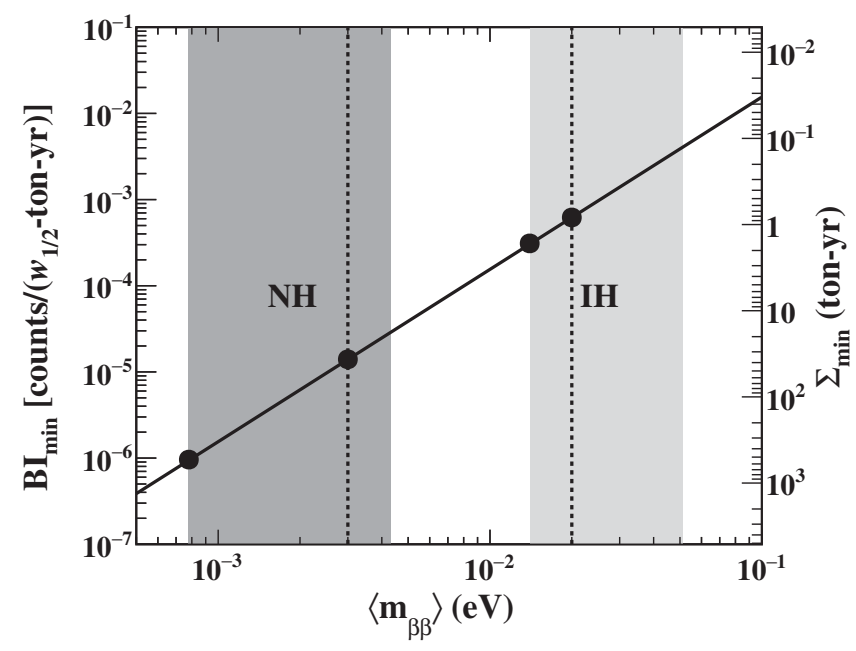

FIG. 7. The variations of the background-free and minimalexposure conditions $\left(\mathrm{BI}_{\min }, \Sigma_{\min }\right)$ at $\mathrm{P}_{50}^{3 \sigma}$ criteria with $\left\langle m_{\beta \beta}\right\rangle$. The IH (NH) bands are defined by $\left(\left\langle m_{\beta \beta}\right\rangle_{-},\left\langle m_{\beta \beta}\right\rangle_{+}\right)$, while their $\left\langle m_{\beta \beta}\right\rangle_{95 \%}$ values are denoted as dotted lines. The black dots correspond to the values listed in the last rows of Table II.
Table II, $\quad \Sigma_{\min }=(0.83,1.7)$ ton-yr at $\mathrm{BI}_{\min } \leq(6.3 \times$ $\left.10^{-4}, 3.1 \times 10^{-4}\right)$ counts $/\left(w_{1 / 2}\right.$-ton-yr $)$ are required to cover $\left(\left\langle m_{\beta \beta}\right\rangle_{95 \%},\left\langle m_{\beta \beta}\right\rangle_{-}\right)^{\mathrm{IH}}$. The corresponding requirements for $\mathrm{NH}$ are $\Sigma_{\min }=(37,550)$ ton-yr at $\mathrm{BI}_{\min } \leq$ $\left(1.4 \times 10^{-5}, 0.96 \times 10^{-6}\right)$ counts $/\left(w_{1 / 2}\right.$-ton-yr $)$. The required $\Sigma_{\min }$ from $\left\langle m_{\beta \beta}\right\rangle_{-}$to $\left\langle m_{\beta \beta}\right\rangle_{95 \%}$ is reduced by $f_{95 \%}=0.49(0.068)$ for $\mathrm{IH}(\mathrm{NH})$.

Alternatively, the $\Sigma=10$ ton-yr target exposure of nextgeneration projects can probe $\left\langle m_{\beta \beta}\right\rangle>\left(5.8 \times 10^{-3}\right) \mathrm{eV}$, approaching $\left\langle m_{\beta \beta}\right\rangle_{+}^{\mathrm{NH}}=\left(4.3 \times 10^{-3}\right) \mathrm{eV}$, when the

(a)

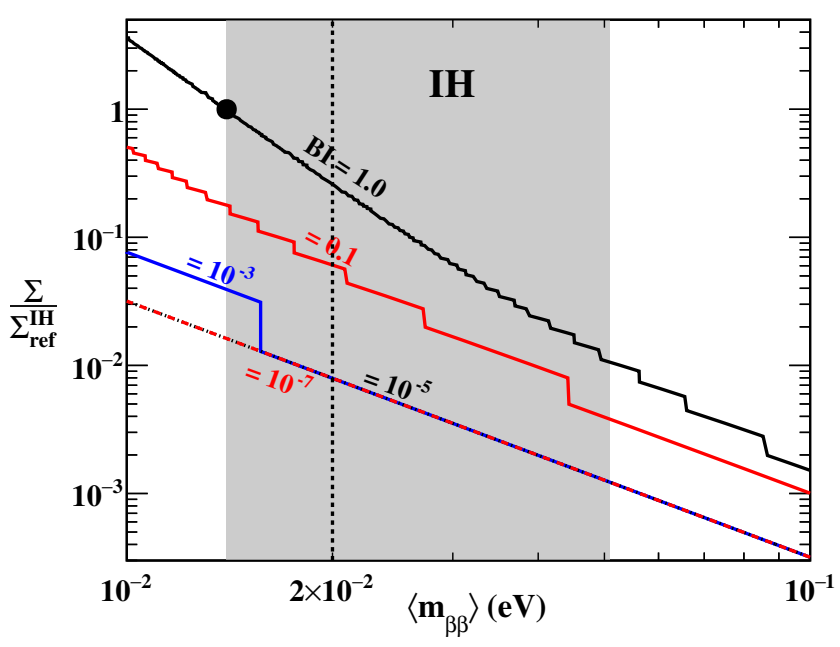

(b)

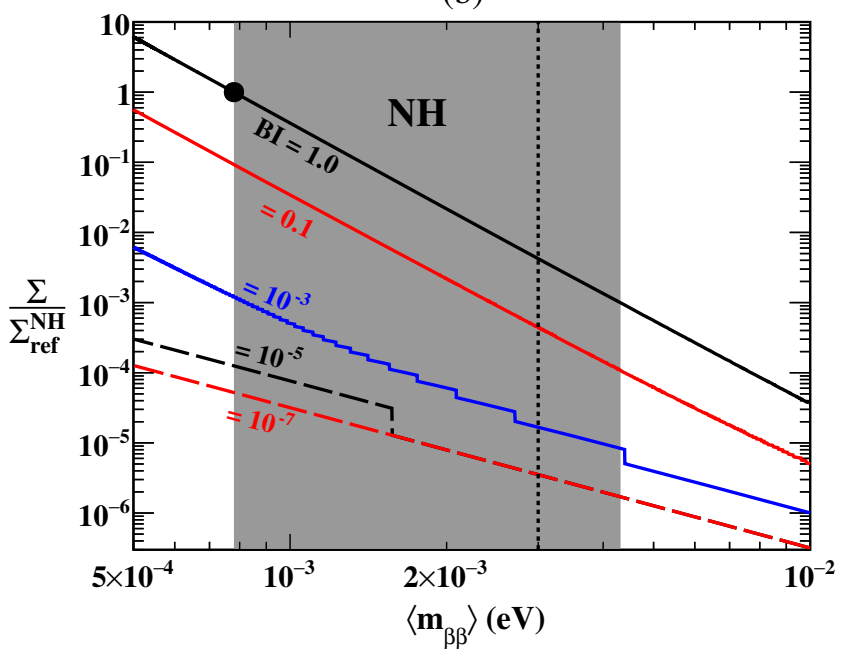

FIG. 8. Fractional reduction of required exposure to probe (a) $\mathrm{IH}$ and (b) $\mathrm{NH}$ as a function of $\left\langle m_{\beta \beta}\right\rangle$ under different $\mathrm{BI}$ background levels in unit of [counts $/\left(w_{1 / 2}\right.$-ton-yr)]. The IH (NH) bands are defined by $\left(\left\langle m_{\beta \beta}\right\rangle_{-},\left\langle m_{\beta \beta}\right\rangle_{+}\right)$, while their $\left\langle m_{\beta \beta}\right\rangle_{95 \%}$ values are denoted as dotted lines. The reference exposures $\Sigma_{\text {ref }}^{\mathrm{IH}(\mathrm{NH})}=110$ ton-yr(11 Mton-yr) to cover $\left\langle m_{\beta \beta}\right\rangle_{-}^{\mathrm{IH}(\mathrm{NH})}$, denoted as black dots, correspond to those required under the current background level of $\mathrm{BI}_{0}=1$ count $/\left(w_{1 / 2}\right.$-ton-yr $)$. 
TABLE III. The range of $\left(\mathrm{S}_{0}, \mathrm{~B}_{0}\right)$ to qualify a positive signal to cover $\left\langle m_{\beta \beta}\right\rangle_{-}$for both IH and NH under $\mathrm{P}_{50}^{3 \sigma}$, given the observed number of events in RoI- $0 \nu \beta \beta$-signals and background are combined but indistinguishable at the event-by-event level. The smaller $\Sigma$ values among the alternatives of RoI $=w_{1 / 2}$ or $w_{3 \sigma}$ are selected. The sixth column shows the required BI which is universal to all $A_{\beta \beta}$. The last column lists the required background specifically for ${ }^{76} \mathrm{Ge}$ normalized to "/(keV-ton-yr)," and the conversion to other isotopes is referred to Eq. (14). The $N_{\text {obs }}^{0 \nu}=1$ row corresponds to the background-free conditions. The BI values follow from Eq. (9).

\begin{tabular}{|c|c|c|c|c|c|c|}
\hline \multicolumn{3}{|c|}{$\begin{array}{c}\text { Counts within } \\
\text { RoI } \\
\end{array}$} & \multirow[t]{2}{*}{$\begin{array}{l}\text { Optimal } \\
\text { RoI }\end{array}$} & \multirow[t]{2}{*}{$\begin{array}{l}\text { Required Exposure } \\
\Sigma \text { (ton-yr) }\end{array}$} & \multirow[t]{2}{*}{$\begin{array}{c}\text { Universal BI } \\
{\left[\text { counts } /\left(w_{1 / 2} \text {-ton-yr }\right)\right]}\end{array}$} & \multirow[t]{2}{*}{$\begin{array}{c}\text { Background } /(\mathrm{keV} \text {-ton-yr }) \\
\text { for }{ }^{76} \mathrm{Ge} \text { at } \Delta=0.12 \%\end{array}$} \\
\hline$N_{\mathrm{obs}}^{0 \nu}$ & $\mathrm{S}_{0}$ & $\mathrm{~B}_{0}$ & & & & \\
\hline \multicolumn{7}{|c|}{ Covering $\left\langle m_{\beta \beta}\right\rangle_{-}$for $\mathrm{IH}:$} \\
\hline 1 & $\geq 0.69$ & $\leq 1.3 \times 10^{-3}$ & $w_{3 \sigma}$ & 1.7 & $\leq 3.1 \times 10^{-4}$ & $\leq 1.2 \times 10^{-4}$ \\
\hline 2 & $\geq 1.6$ & $\leq 5.2 \times 10^{-2}$ & $w_{3 \sigma}$ & 4.0 & $\leq 5.2 \times 10^{-3}$ & $\leq 2.1 \times 10^{-3}$ \\
\hline 3 & $\geq 2.5$ & $\leq 0.21$ & $w_{3 \sigma}$ & 6.0 & $\leq 1.4 \times 10^{-2}$ & $\leq 5.6 \times 10^{-3}$ \\
\hline 4 & $\geq 3.2$ & $\leq 0.45$ & $w_{1 / 2}$ & 10 & $\leq 4.3 \times 10^{-2}$ & $\leq 1.8 \times 10^{-2}$ \\
\hline 5 & $\geq 3.9$ & $\leq 0.77$ & $w_{1 / 2}$ & 13 & $\leq 6.1 \times 10^{-2}$ & $\leq 2.4 \times 10^{-2}$ \\
\hline 10 & $\geq 6.6$ & $\leq 3.1$ & $w_{1 / 2}$ & 21 & $\leq 0.14$ & $\leq 6.0 \times 10^{-2}$ \\
\hline \multicolumn{7}{|c|}{ Covering $\left\langle m_{\beta \beta}\right\rangle_{-}$for $\mathrm{NH}$ : } \\
\hline 1 & $\geq 0.69$ & $\leq 1.3 \times 10^{-3}$ & $w_{3 \sigma}$ & $5.5 \times 10^{2}$ & $\leq 0.96 \times 10^{-6}$ & $\leq 0.38 \times 10^{-6}$ \\
\hline 2 & $\geq 1.6$ & $\leq 5.2 \times 10^{-2}$ & $w_{3 \sigma}$ & $1.3 \times 10^{3}$ & $\leq 1.6 \times 10^{-5}$ & $\leq 6.4 \times 10^{-6}$ \\
\hline 3 & $\geq 2.5$ & $\leq 0.21$ & $w_{3 \sigma}$ & $2.0 \times 10^{3}$ & $\leq 4.0 \times 10^{-5}$ & $\leq 1.7 \times 10^{-5}$ \\
\hline 4 & $\geq 3.2$ & $\leq 0.45$ & $w_{1 / 2}$ & $3.4 \times 10^{3}$ & $\leq 1.3 \times 10^{-4}$ & $\leq 5.4 \times 10^{-5}$ \\
\hline 5 & $\geq 3.9$ & $\leq 0.77$ & $w_{1 / 2}$ & $4.2 \times 10^{3}$ & $\leq 1.8 \times 10^{-4}$ & $\leq 7.5 \times 10^{-5}$ \\
\hline 10 & $\geq 6.6$ & $\leq 3.1$ & $w_{1 / 2}$ & $7.0 \times 10^{3}$ & $\leq 4.4 \times 10^{-4}$ & $\leq 1.8 \times 10^{-4}$ \\
\hline
\end{tabular}

background-free condition $\mathrm{BI}_{\min }<5.1 \times 10^{-5}$ counts/ $\left(w_{1 / 2}\right.$-ton-yr $)$ is achieved.

The interplay between fractional reduction of BI and $\Sigma$ relative to $\mathrm{BI}_{0}$ and $\Sigma_{\text {ref }}^{\mathrm{IH}(\mathrm{NH})}$ to cover $\left\langle m_{\beta \beta}\right\rangle_{95 \%}$ and $\left\langle m_{\beta \beta}\right\rangle_{-}$in $\mathrm{IH}(\mathrm{NH})$ is depicted in Fig. 6(b). Background-free conditions require additional BI suppression by factors of $3.1 \times 10^{-4}\left(0.96 \times 10^{-6}\right)$, to cover $\left\langle m_{\beta \beta}\right\rangle_{-}^{\mathrm{IH}(\mathrm{NH})}$ in which cases $\Sigma$ can be reduced by factors of $0.016\left(5 \times 10^{-5}\right)$. The shaded regions match those of Fig. 6(a) in displaying the preferred hardware specification space.

The impact of background suppression to the required exposure is increasingly enhanced as smaller values of $\left\langle m_{\beta \beta}\right\rangle$ are probed. This is illustrated in Figs. 8(a) and 8(b) which display the reduction fraction in $\Sigma$ relative to $\Sigma_{\text {ref }}^{\mathrm{IH}(\mathrm{NH})}$ at different background levels. For instance, the suppression of BI from 1 to $10^{-3}$ counts $/\left(w_{1 / 2}\right.$-ton-yr) will contribute to the reduction of $\Sigma$ from $\Sigma=(27,110)$ ton-yr to $(1.1,4.1)$ ton-yr and from $\Sigma=(44,11000)$ kton - yr to $(0.17,13)$ kton-yr to cover $\left(\left\langle m_{\beta \beta}\right\rangle_{95 \%},\left\langle m_{\beta \beta}\right\rangle_{-}\right)^{\mathrm{IH}}$ and $\left(\left\langle m_{\beta \beta}\right\rangle_{95 \%},\left\langle m_{\beta \beta}\right\rangle_{-}\right)^{\mathrm{NH}}$, respectively.

In realistic experiments, signals and background are indistinguishable at the event-by-event level. The expected average background $\mathrm{B}_{0}$ and the observed event counts (an integer) in the RoI are the known quantities. They can be used to assess whether a signal is "established" under certain criteria like $\mathrm{P}_{50}^{3 \sigma}$. Listed in Table III are the required ranges on $\left(\mathrm{S}_{0}, \mathrm{~B}_{0}\right)$ to qualify positive signals given the number of observed events. The first row corresponds to the background-free condition, in which one single event is sufficient to establish a signal. Accordingly, the (BI, $\Sigma$ ) values match the entries in the last rows of Table II, and are displayed in Fig. 7.

Results of Table III, apply generically to all $A_{\beta \beta}$ except those for the last column when background is expressed in the "/(keV-ton-yr)" unit. The values are specific for ${ }^{76} \mathrm{Ge}$, where the best published $\Delta\left({ }^{76} \mathrm{Ge}\right)=0.12 \%$ of the MJD experiment [15] is adopted as input. The background requirements for other $A_{\beta \beta}$ can be derived via

$$
\begin{gathered}
\frac{[\text { Background } /(\text { keV-ton-yr })]\left(A_{\beta \beta}\right)}{[\text { Background } /(\mathrm{keV} \text {-ton-yr })]\left({ }^{76} \mathrm{Ge}\right)} \\
\quad=\left[\frac{\Delta\left({ }^{76} \mathrm{Ge}\right)}{\Delta\left(A_{\beta \beta}\right)}\right]\left[\frac{Q_{\beta \beta}\left({ }^{76} \mathrm{Ge}\right)}{Q_{\beta \beta}\left(A_{\beta \beta}\right)}\right] .
\end{gathered}
$$

\section{B. Limiting irreducible background}

It is instructive and important to quantify the interplay between various irreducible background channels to the required exposure. In particular, one such irreducible background is the Standard-Model-allowed $2 \nu \beta \beta$

$$
{ }_{Z}^{N} A_{\beta \beta} \rightarrow{ }_{Z+2}^{N-2} A+2 e^{-}+2 \bar{\nu}_{e} .
$$

The contamination levels to $0 \nu \beta \beta$ at the $Q_{\beta \beta}$-associated RoI depend on its half-life $\left(T_{1 / 2}^{2 \nu}\right)$ and the detector resolution. A worse resolution (larger $\Delta$ ) implies a larger RoI range to 


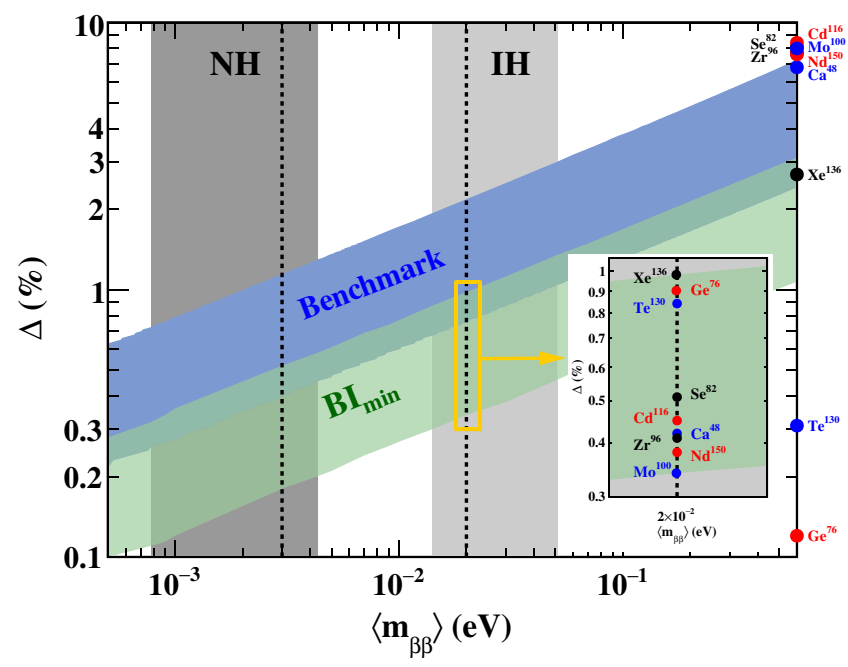

FIG. 9. Variations of the required $\Delta$ with $\left\langle m_{\beta \beta}\right\rangle$ such that $2 \nu \beta \beta$ background within $\mathrm{RoI}=w_{3 \sigma}$ would contribute less than those specified by the benchmark $\left[1\right.$ count $\left./\left(w_{1 / 2}-\Sigma\right)\right]$ level and the background-free $\left(\mathrm{BI}_{\min }\right)$ condition of Fig. 7 as a function of $\left\langle m_{\beta \beta}\right\rangle$. The relative locations of $A_{\beta \beta}$ within both bands are depicted in the inset, using the $\mathrm{BI}_{\min }$ band as illustration. The best achieved $\Delta$ 's in past and ongoing experiments are displayed at the right vertical axis.

search for $0 \nu \beta \beta$ signals, and therefore a higher probability of having background events from the $2 \nu \beta \beta$ spectral tail.

Depicted in Fig. 9 are variations of the required $\Delta$ with $\left\langle m_{\beta \beta}\right\rangle$ such that $2 \nu \beta \beta$ background within RoI $=w_{3 \sigma}$ would contribute less than the BI values specified by the benchmark and background-free conditions. The finite width of the band is a consequence of the spread of measured $T_{1 / 2}^{2 \nu}$ $[2,13]$. Faster $2 \nu \beta \beta$ rates typically require better detector resolution to define smaller RoI. The relative locations for different $A_{\beta \beta}$ within the bands are depicted in the inset.

Listed in Table IV are the required ranges of $\Delta$ to cover $\left\langle m_{\beta \beta}\right\rangle_{95 \%}^{\mathrm{IH}(\mathrm{NH})}$ and $\left\langle m_{\beta \beta}\right\rangle_{-}^{\mathrm{IH}(\mathrm{NH})}$. In particular, the required resolutions to cover $\left\langle m_{\beta \beta}\right\rangle_{-}$for $\mathrm{IH}$ and $\mathrm{NH}$ under background-free conditions are $\Delta \leq(0.3-0.9) \%$ and $\Delta \leq$ $(0.1-0.4) \%$, respectively. The best achieved $\Delta$ for past and ongoing $0 \nu \beta \beta$ experiments $[2,15,16]$ are included in Table IV and depicted on the right vertical axis of Fig. 9 for comparison. In particular, the best published $\Delta\left({ }^{76} \mathrm{Ge}\right)=$ $0.12 \%$ [15] corresponds to an irreducible $2 \nu \beta \beta$ background contribution of $\mathrm{BI}<6 \times 10^{-10}$ counts/( $w_{1 / 2}$-ton-yr $)$. This provides a comfortable margin relative to that which satisfies the background-free conditions for $\left\langle m_{\beta \beta}\right\rangle_{-}^{\mathrm{NH}}$ at $\mathrm{BI} \leq 0.96 \times 10^{-6}$ counts $/\left(w_{1 / 2}\right.$-ton-yr $)$.

\section{SUMMARY AND PROSPECTS}

As current neutrino oscillation experiments reveal a preference of $\mathrm{NH}$, the strategy of scaling the summit of $0 \nu \beta \beta$ should take this genuine possibility into account.

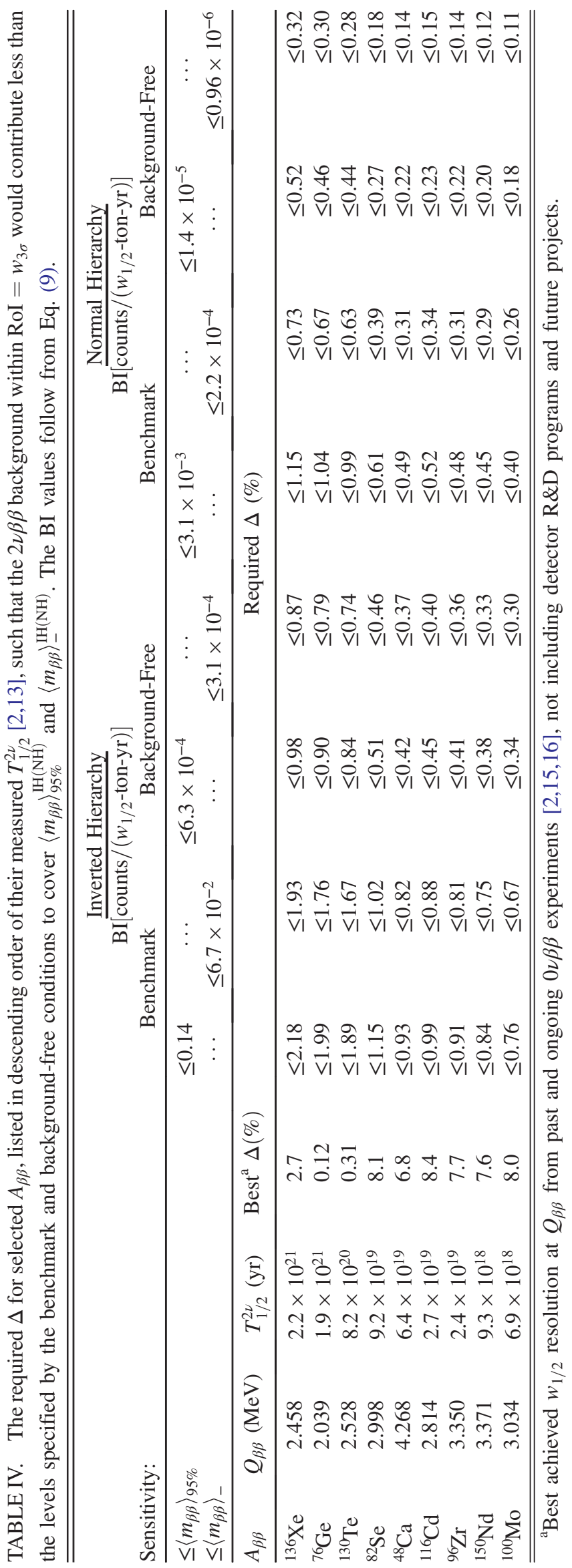


This work studies the relation between the two main factors in improving experimental sensitivities: (BI, $\Sigma$ ). We recall that the presented results are derived with certain input parameter choice: IA $=100 \%, \varepsilon_{\text {expt }}=100 \%$, and $g_{A}=1.27$, and that $0 \nu \beta \beta$ is driven by the Majorana neutrino mass terms via the mass mechanism while the signal-to-background analysis is based on counting experiments without exploiting the spectral shape information at this stage.

Advancing towards ND-NH to cover $\left\langle m_{\beta \beta}\right\rangle_{-}^{\mathrm{NH}}$ will require large and costly exposure. An unrealistic $\mathcal{O}(10)$ Mton-yr enriched target mass is necessary at the current best achieved background level $\mathrm{BI}_{0} \sim 1$ count/( $w_{1 / 2}$-ton-yr). Reduction of BI will be playing increasingly significant, if not determining, roles in shaping future $0 \nu \beta \beta$ projects.

For instance, following Table II, background-free conditions for $\left\langle m_{\beta \beta}\right\rangle_{-}^{\mathrm{NH}}$ correspond to additional background suppression from the current best $\mathrm{BI}_{0} \sim 1$ count/ $\left(w_{1 / 2}\right.$-ton-yr $)$ and benchmark [1 count $\left./\left(w_{1 / 2}-\Sigma\right)\right]$ levels by factors of $\left(0.96 \times 10^{-6}\right)$ and $\left(4.4 \times 10^{-3}\right)$, respectively. This would reduce the required $\Sigma$ from 11 Mton-yr and 4600 ton-yr, respectively, to 550 ton-yr. The corresponding minimal exposure to cover $\left\langle m_{\beta \beta}\right\rangle_{95 \%}^{\mathrm{NH}}$ is $\Sigma_{\min } \sim 37$ ton-yr, which is only a modest factor beyond the goals of nextgeneration experiments [14]. The pursuit of background towards $\mathrm{BI} \sim \mathcal{O}\left(10^{-6}\right)$ counts $/\left(w_{1 / 2}\right.$-ton-yr $)$ to probe $\mathrm{ND}-\mathrm{NH}$, while challenging, is highly investment effective, as it is equivalent to reduction of $\Sigma$ by $\mathcal{O}(10)$ Mton-yr and $\mathcal{O}(1)$ kton-yr relative to those required for the current best and benchmark background levels, respectively.

This article serves to quantify the merits of background reduction in $0 \nu \beta \beta$ experiments, but does not attempt to address the experimental issues on how to realize the feat and how to demonstrate that the suppression factors are achieved when experiments are constructed. We project that the continuous intense efforts and ingenuities from the experimentalists worldwide, with motivations reinforced by the increasing equivalent "market" values, will be able to meet the challenges.

Boosting $\Sigma$ involves mostly in the accumulation of enriched $A_{\beta \beta}$ isotopes and turning these into operating detectors. These processes are confined to relatively few locations and small communities of expertise. The room for development which may overcome the known hurdles is limited. Suppression of the $0 \nu \beta \beta$ experimental background, on the other hand, would be the tasks of mobilizing and coordinating the efforts of a large pool of expertise. It is related to the advances in diverse disciplines from novel materials to chemistry processing to trace measurement techniques. Research programs on many subjects requiring low-background techniques may contribute to-and benefit from-the advances. There would be strong potentials of technological breakthroughs and innovative ideas as the sensitivity goals are pursued.

Signal efficiencies are also increasingly costly as sensitivities advance towards ND-NH. For instance, at $\Sigma_{\min }=$ 550 ton-yr to cover $\left\langle m_{\beta \beta}\right\rangle_{-}^{\mathrm{NH}}$, a high $90 \%$ efficiency to certain selection criterion corresponds to discarding data of $\mathcal{O}(10)$ ton-yr strength - already an order of magnitude larger than the combined exposure of all $0 \nu \beta \beta$ experiments. It follows that background suppression would preferably be attended at the root level - that radioactive contaminations are suppressed to start with, rather than relying on special signatures and software selection algorithms to identify them.

The next generation of $0 \nu \beta \beta$ experiments would cover $\left\langle m_{\beta \beta}\right\rangle_{-}^{\dagger \mathrm{H}}$. In addition, they should be able to explore the strategies and demonstrate sufficient margins to advance towards $\left\langle m_{\beta \beta}\right\rangle_{-}^{\mathrm{NH}}$. A significant merit would be to have no irreducible background before reaching the $\mathrm{BI} \sim$ $\mathcal{O}\left(10^{-6}\right)$ counts/( $w_{1 / 2}$-ton-yr) background-free configuration. The detector requirements to achieve this for $2 \nu \beta \beta$ are summarized in Fig. 9 and Table IV. Detailed studies of this background as well as other channels like those due to residual cosmogenic radioactivity and long-lived radioactive isotopes are themes of our ongoing research efforts.

\section{ACKNOWLEDGMENTS}

This work is supported by the Academia Sinica Principal Investigator Award AS-IA-106-M02, Contracts No. 1042112-M-259-004-MY3 and No. 107-2119-M-001-028MY3 from the Ministry of Science and Technology, Taiwan, and No. 2017-ECP2 from the National Center of Theoretical Sciences, Taiwan.
[1] M. Tanabashi et al. (Particle Data Group), Phys. Rev. D 98, 030001 (2018), and in particular, Review 14, p. 251 by K. Nakamura and S. T. Petcov, and references therein.

[2] S. Dell'Oro et al., Adv. High Energy Phys. 2016, 2162659 (2016); L. Cardani, SciPost Phys. Proc. 1, 024 (2019), and references therein; M. J. Dolinski, A. W. P. Poon, and W. Rodejohann, Annu. Rev. Nucl. Part. Sci. 69, 219 (2019).

[3] S. Bilenky, Nucl. Phys. B908, 2 (2016).

[4] T. Nakaya and R. K. Plunkett, New J. Phys. 18, 015009 (2016); F. Simpson, R. Jimenez, C. Pena-Garay, and L. Verde, J. Cosmol. Astropart. Phys. 06 (2017) 029; I. Eseban, 
M. C. Gonzalez-Garcia, A. Hernandez-Cabezudo, M. Maltoni, and T. Schwetz, J. High Energy Phys. 01 (2019) 106; M. G. Aartsen et al., arXiv:1902.07771.

[5] M. Tanabashi et al. (Particle Data Group), Phys. Rev. D 98, 030001 (2018), and in particular, Review 25, p. 390 by J. Lesgourgues and L. Verde, and references therein; N. Aghanim et al., arXiv:1807.06209v1.

[6] A. Dueck, W. Rodejohann, and K. Zuber, Phys. Rev. D 83, 113010 (2011).

[7] R. G. H. Robertson, Mod. Phys. Lett. A 28, 1350021 (2013).

[8] J. Barea, J. Kotila, and F. Iachello, Phys. Rev. C 87, 014315 (2013); S. DellOro, S. Marcocci, and F. Vissani, Phys. Rev. D 90, 033005 (2014).

[9] J. Kotila and F. Iachello, Phys. Rev. C 85, 034316 (2012).

[10] J. Engel, and J. Menéndez, Rep. Prog. Phys. 80, 046301 (2017).
[11] G. Benato, Eur. Phys. J. C 75, 563 (2015); A. Caldwell, A. Merle, O. Schulz, and M. Totzauer, Phys. Rev. D 96, 073001 (2017); M. Agostini, G. Benato, and J. A. Detwiler, Phys. Rev. D 96, 053001 (2017); A. Di Iura and D. Meloni, Nucl. Phys. B921, 829 (2017).

[12] V. A. Rodin, A. Faessler, F. Šimkovic, and P. Vogel, Nucl. Phys. A766, 107 (2006); J. Barea, J. Kotila, and F. Iachello, Phys. Rev. C 91, 034304 (2015).

[13] M. Agostini et al., Nature (London) 544, 47 (2017); Phys. Rev. Lett. 120, 132503 (2018); Science 365, 1445 (2019).

[14] G. Wang et al., arXiv:1504.03599; N. Abgrall et al., AIP Conf. Proc. 1894, 020027 (2017); J. B. Albert et al., Phys. Rev. C 97, 065503 (2018).

[15] C. E. Aalseth et al., Phys. Rev. Lett. 120, 132502 (2018).

[16] G. Anton et al., Phys. Rev. Lett. 123, 161802 (2019). 